\title{
Road Traffic Models using Petri Nets and Minplus Algebra
}

\author{
Nadir Farhi, Maurice Goursat, and Jean-Pierre Quadrat \\ INRIA - Rocquencourt, Domaine de Voluceau \\ 78153 Le Chesnay, Cedex, France.
}

\begin{abstract}
We present in this paper a road traffic model based on Petri nets and minplus algebra in order to study the fundamental traffic diagram of regular city (american like city). The obtained dynamic is simulated using an extension of the maxplus toolbox of Scilab able to simulate general Petri nets with negative multipliers. The Petri net model corresponding to an intersection with traffic lights is described first. Then the construction of the regular city dynamics is done. An algebra point of view is used. Systems are seen as operators corresponding to Petri nets with named inputs and outputs which can be represented by a set of matrices in standard and maxplus algebra. On such systems standard operations parallel series composition feedback and contraction (a feedback generalization) are defined. Then a regular city dynamic is obtained by combining three elementary systems with these combinators. The fundamental diagrams of some regular cities are shown to illustrate the possibilities of the toolbox.
\end{abstract}

\section{Introduction}

Two modeling approaches of road traffic can distinguished : - the macroscopic point of view where the traffic is seen as a gas with a comportment law given by a diagram called the fundamental traffic diagram, - the microscopic point of view where each individual vehicle with a specific comportment is distinguished. The cellular automata approach is a microscopic one with very simplified individual comportment where large simulations are done to make macroscopic laws stand out. In this paper, we present a microscopic model based on Petri net [8] whose dynamic is written using minplus algebra [1]. Our approach is microscopic, not far from the cellular automata approach $[11,12]$. As in statistical physics $[10,14-$ 16], we study the individual movement of vehicles to derive macroscopic laws. The effectiveness of this type of model in determining fundamental traffic diagrams has been shown on a system without intersection [9].

First, we show that crossings with traffic lights can be modeled using time invariant Petri nets with negative multiplicities on some arcs. Indeed with negative multiplicities we can model tokens staying only a finite time in a place. This property is necessary to be able to represent the phases of the traffic light by tokens. An alternative way of modeling is to consider that the Petri net change with the phases but in this case the Petri nets become time variant.

Second, we use a system point of view to build a large Petri net representing a regular city. A regular city is an american like, one-way parallel streets regularly spaced, one in a direction the next one in the other direction. 
To build the dynamics of a regular city we introduced a new system theory. Systems are seen as operators corresponding to Petri nets with named inputs and outputs. The dynamics of such systems can be defined by three standard matrices and three maxplus algebra matrices. On such systems some operations can be defined parallel and series composition and different sort of feedbacks. Then a regular city dynamic is obtained by combining three elementary systems with these combinators. The dynamics of free choice Petri nets with negative multiplicites is also introduced, it is useful to model crossings with control lights.

Third, by simulation of the built system the fundamental diagrams of regular cities can be computed using the maxplus algebra toolbox of Scilab. Some diagram examples are given. On these diagram appears clearly three phases which have been previously commented on simple systems see [2]-[3].

\section{Minplus algebra}

The structure $\mathbb{R}_{\text {min }}=(\mathbb{R} \cup\{+\infty\}, \oplus, \otimes)$ is defined by the set $\mathbb{R} \cup\{+\infty\}$ endowed with the operations min (denoted by $\oplus$, called minplus sum) and + (denoted by $\otimes$, called minplus product). The element $\varepsilon=+\infty$ is the zero element, it satisfies $\varepsilon \oplus x=x$ and is absorbing $\varepsilon \otimes x=\varepsilon$. The element $e=0$ is the unity, it satisfies $e \otimes x=x$. The main difference with respect to the conventional algebra is the idempotency of the addition $x \oplus x=x$ and the fact that addition cannot be simplified, that is: $a \oplus b=c \oplus b \nRightarrow a=c$. It is called minplus algebra.

This minplus structure on scalars induces an idempotent semiring structure on $m \times m$ square matrices with the element-wise minimum and matrix product defined by $(A \otimes B)_{i k}=\min _{j}\left(A_{i j}+B_{j k}\right)$, where the zero and unit matrices are still denoted by $\varepsilon$ and $e$.

Event Graph (Petri nets (see next section) with places having only one input and one output arcs) are linear dynamical system in this algebra and can be represented by a triple $(A, B, C)$ of minplus matrices. The throughput of the event graph is then given by the minplus eigenvalue of the $A$ matrix see [1].

Traffic on a circular road can be described in term of event graph and then the fundamental diagram giving the average flow as a function of the car density correspond to compute the eigen value of $A$ as a function of the number of tokens. Then, the graph interpretation of the eigen value of the matrix $A$ gives explicitly the fundamental diagram see [9].

\section{Petri Net Dynamics}

A Petri net $\mathcal{N}$ is a graph with two set of nodes: the transitions $\mathcal{Q}$ (with $Q$ elements) and the places $\mathcal{P}$ (with $P$ elements) and two sorts of arcs, the synchronization arcs (from a place to a transition) and the production arcs (from a transition to a place).

A minplus $P \times Q$ matrix $D$, called synchronization (decision) matrix is associated to the synchronization arcs. $D_{p q}=a_{p}$ if there exists an arc from the place 
$p \in \mathcal{P}$ to the transition $q \in \mathcal{Q}$, and $D_{p q}=\varepsilon$ elsewhere, where $a_{p}$ is the initial marking and is represented graphically by the tokens in the places. We suppose here that the sojourn time in all the places is one time unit ${ }^{1}$.

A standard algebra $Q \times P$ matrix $H$, called production (hazard) matrix is associated with the production arcs. It is defined by $H_{q p}=m_{q p}$ if there exists an $\operatorname{arc}$ from $q$ to $p$, and 0 elsewhere, where $m_{q p}$ is the multiplicity of the arc.

Therefore a Petri net is characterized by the quadruple :

$$
(\mathcal{P}, \mathcal{Q}, H, D) .
$$

It is a dynamic system in which the token evolution is partially defined by the transition firings saying that a transition can fire as soon as all its upstream places contain at least a token having stayed at least one unit of time. When a transition fires, it generates a number of tokens in each downstream place equal to the multiplicity of the arc joining the transition to the place.

In the case of a deterministic Petri net, where all the places have only one arc downstream, the dynamic is well defined, that is, there is no token consuming conflict between the downstream transitions ${ }^{2}$. Then, denoting $Q=\left(Q_{q}^{k}\right)_{q \in \mathcal{Q}, k \in \mathbb{N}}$ the vector of sequences of cumulated firing numbers of transitions and $P=$ $\left(P_{p}^{k}\right)_{p \in \mathcal{P}, k \in \mathbb{N}}$ the vector of sequences of cumulated tokens numbers arrived in the places we have :

$$
\left[\begin{array}{l}
P^{k+1} \\
Q^{k+1}
\end{array}\right]=\left[\begin{array}{cc}
0 & H \\
D & \varepsilon
\end{array}\right] \otimes\left[\begin{array}{c}
P^{k+1} \\
Q^{k}
\end{array}\right] \triangleq\left[\begin{array}{c}
H Q^{k} \\
D \otimes P^{k+1}
\end{array}\right]
$$

From this dynamics we can deduce the dynamics transition by eliminating the places variables and the dynamics of transition by eliminating the transition vraiables :

$$
Q^{k+1}=D \otimes\left(H Q^{k}\right), \quad P^{k+1}=H\left(D \otimes P^{k}\right) .
$$

In the case of event graphs, particular deterministic Petri nets where all the multiplicities $m_{q p}$ are equal to 1 and all the places have exactly one arc upstream, the dynamics is linear in the minplus sense. It is :

$$
Q^{k+1}=A \otimes Q^{k}
$$

where $A_{q q^{\prime}}=a_{p}$ with $p$ the unique place connected to the transitions $q$ and $q^{\prime}$.

\subsection{Input-Output Petri nets}

In this section, we partition the transition $(\mathcal{V}, \mathcal{Q}, \mathcal{Z})[$ resp. the places $(\mathcal{U}, \mathcal{P}, \mathcal{Y})]$ in three sets : - the inputs, - the states, - the outputs. The inputs are the transitions

\footnotetext{
${ }^{1}$ When different integer sojourn times are considered, an equivalent Petri net with a unique sojourn time can obtained by adding places and transitions and solving the implicit relations.

${ }^{2}$ In the nondeterministic case, we have to precise the rules which resolve the conflicts by, for example, giving priorities to the consuming transitions or by imposing ratios to be respected. As soon as this rules are added, the initial nondeterministic Petri net becomes a deterministic one.
} 
[resp. places] without upstream arcs. The outputs are the ones without output arcs. Then the dynamics can be rewritten :

$$
\left[\begin{array}{l}
P^{k+1} \\
Q^{k+1} \\
Y^{k+1} \\
Z^{k+1}
\end{array}\right]=\left[\begin{array}{llll}
0 & A & 0 & B \\
C & \varepsilon & D & \varepsilon \\
0 & E & 0 & 0 \\
F & \varepsilon & \varepsilon & \varepsilon
\end{array}\right] \otimes\left[\begin{array}{c}
P^{k+1} \\
Q^{k} \\
U^{k+1} \\
V^{k}
\end{array}\right] \triangleq\left[\begin{array}{c}
A Q^{k}+B V^{k} \\
C \otimes P^{k+1} \oplus D \otimes U^{k+1} \\
E Q^{k} \\
F \otimes P^{k+1}
\end{array}\right]
$$

This dynamics denoted $S$, defined by the matrices $(A, \cdots, F)$, associates to the input signals $\left(U^{k}, V^{k}\right)_{k \in \mathbb{N}}$ the output signals $\left(Y^{k}, Z^{k}\right)_{k \in \mathbb{N}}:(Y, Z)=S(U, V)$.

On these systems we can define operations :

- Parallel Composition. Given two systems $S_{1}$ and $S_{2}$ we define the system $S=S_{1} \boxplus S_{2}$ obtained by putting in common the entries and by adding ${ }^{3}$ the output places. The dynamics of $S$ is defined by :

$$
\begin{gathered}
A=\left[\begin{array}{cc}
A_{1} & 0 \\
0 & A_{2}
\end{array}\right], B=\left[\begin{array}{l}
B_{1} \\
B_{2}
\end{array}\right], C=\left[\begin{array}{cc}
C_{1} & \varepsilon \\
\varepsilon & C_{2}
\end{array}\right], D=\left[\begin{array}{l}
D_{1} \\
D_{2}
\end{array}\right], \\
E=\left[E_{1} E_{2}\right], F=\left[\begin{array}{ll}
F_{1} & F_{2}
\end{array}\right] .
\end{gathered}
$$

- Series Composition. Given two systems $S_{1}$ and $S_{2}$ we define the system $S=S_{1} \otimes S_{2}$ obtained by composition of the two systems $S(U, V)=$ $S^{1}\left(S^{2}(U, V)\right)$. The dynamics is defined by :

$$
\begin{gathered}
A=\left[\begin{array}{ccc}
A_{1} & 0 & B_{1} \\
0 & A_{2} & 0 \\
0 & E_{2} & 0
\end{array}\right], B=\left[\begin{array}{c}
0 \\
B_{2} \\
0
\end{array}\right], C=\left[\begin{array}{ccc}
C_{1} & \varepsilon & D_{1} \\
\varepsilon & C_{2} & \varepsilon \\
\varepsilon & F_{2} & \varepsilon
\end{array}\right], D=\left[\begin{array}{c}
\varepsilon \\
D_{2} \\
\varepsilon
\end{array}\right], \\
E=\left[\begin{array}{lll}
E_{1} & 0 & 0
\end{array}\right], F=\left[\begin{array}{lll}
F_{1} & \varepsilon
\end{array}\right] .
\end{gathered}
$$

- Feedback. Given a system $S$, we define the feedback system $S^{\boxplus}$ as the solution in $(Y, Z)$ of $(Y, Z)=S((U, V) \boxplus(Y, Z))=\left(S^{1}(U \oplus Y), S^{2}(V+Z)\right)$. Its dynamics is defined by :

$$
\begin{gathered}
A^{\boxplus}=\left[\begin{array}{ll}
A & B \\
E & 0
\end{array}\right], B^{\boxplus}=\left[\begin{array}{c}
B \\
0
\end{array}\right], C^{\boxplus}=\left[\begin{array}{ll}
C & D \\
F & \varepsilon
\end{array}\right], D^{\boxplus}=\left[\begin{array}{c}
D \\
\varepsilon
\end{array}\right], \\
E^{\boxplus}=\left[\begin{array}{ll}
E & 0
\end{array}\right], F^{\boxplus}=\left[\begin{array}{ll}
F & \varepsilon
\end{array}\right] .
\end{gathered}
$$

- Contraction. To define contraction we have first to partition the inputs (denoted in the case of a partition in two set by $\left(i, i^{\prime}\right)$ ) and the outputs (dented $\left.\left(o, o^{\prime}\right)\right)$ of a system. Then a system $S$ with the previous partition of inputs and outputs is denoted $S_{o o^{\prime}}^{i i^{\prime}}$.

\footnotetext{
${ }^{3}$ The output-places are added in the standard algebra and the transition in the minplus algebra.
} 
Given two partitioned systems $S_{o o^{\prime}}^{i i^{\prime}}$ and $\bar{S}_{i^{\prime} o^{\prime \prime}}^{i^{\prime \prime}}$ we define the contracted system $\hat{S}_{o o^{\prime \prime}}^{i i^{\prime \prime}} \triangleq S_{o o^{\prime}}^{i i^{\prime}} \bar{S}_{i^{\prime} o^{\prime \prime}}^{o^{\prime} i^{\prime \prime}}$ as the solution in $Y, Y^{\prime \prime}, Z, Z^{\prime \prime}$ of the system :

$$
\left\{\begin{array}{l}
\left(Y Y^{\prime}, Z Z^{\prime}\right)=S\left(U U^{\prime}, V V^{\prime}\right), \\
\left(U^{\prime} Y^{\prime \prime}, V^{\prime} Z^{\prime \prime}\right)=\bar{S}\left(Y^{\prime} U^{\prime \prime}, Z^{\prime} V^{\prime \prime}\right),
\end{array}\right.
$$

where the partition of the input places associated to the input partition $\left(i, i^{\prime}\right)$ is denoted $U U^{\prime}$ with the analogous notations for the outputs $Y Y^{\prime}$ and for the input and output transitions $V V^{\prime}$ and $Z Z^{\prime}$. The dynamic of $\hat{S}$ is defined by :

$$
\begin{gathered}
\hat{A}=\left[\begin{array}{cccc}
A & 0 & 0 & B^{\prime} \\
0 & \bar{A} & \bar{B}^{\prime} & 0 \\
E^{\prime} & 0 & 0 & 0 \\
0 & \bar{E}^{\prime} & 0 & 0
\end{array}\right], \hat{B}=\left[\begin{array}{cc}
B & 0 \\
0 & \bar{B}^{\prime \prime} \\
0 & 0 \\
0 & 0
\end{array}\right], \hat{C}=\left[\begin{array}{cccc}
C & \varepsilon & \varepsilon & D^{\prime} \\
\varepsilon & \bar{C} & \bar{D}^{\prime} & \varepsilon \\
F^{\prime} & \varepsilon & \varepsilon & \varepsilon \\
\varepsilon & \bar{F}^{\prime} & \varepsilon & \varepsilon
\end{array}\right], \hat{D}=\left[\begin{array}{cc}
D & \varepsilon \\
\varepsilon & \bar{D}^{\prime \prime} \\
\varepsilon & \varepsilon \\
\varepsilon & \varepsilon
\end{array}\right], \\
\hat{E}=\left[\begin{array}{cccc}
E & 0 & 0 & 0 \\
0 & \bar{E}^{\prime \prime} & 0 & 0
\end{array}\right], \hat{F}=\left[\begin{array}{cccc}
F & \varepsilon & \varepsilon & \varepsilon \\
\varepsilon & \bar{F}^{\prime \prime} & \varepsilon & \varepsilon
\end{array}\right],
\end{gathered}
$$

Where the input matrices of $S$ and $\bar{S}$ have been partitioned in :

$$
\left[B B^{\prime}\right],\left[\bar{B}^{\prime} \bar{B}^{\prime \prime}\right],\left[D D^{\prime}\right],\left[\bar{D}^{\prime} \bar{D}^{\prime \prime}\right],
$$

and the output matrices have been partitioned in :

$$
\left[\begin{array}{c}
E \\
E^{\prime}
\end{array}\right],\left[\begin{array}{c}
\bar{E}^{\prime} \\
\bar{E}^{\prime \prime}
\end{array}\right],\left[\begin{array}{c}
F \\
F^{\prime}
\end{array}\right],\left[\begin{array}{c}
\bar{F}^{\prime} \\
\bar{F}^{\prime \prime}
\end{array}\right]
$$

\subsection{Some Simple Input Output Petri Nets}

It is clear that these systems can be specialized in the standard linear systems or in maxplus linear systems. There is also other classes where composition operators become simpler for example the system ${ }^{4}$ :

$$
\left\{\begin{array}{l}
Q^{k+1}=C \otimes\left(A Q^{k}+B V^{k}\right), \\
Z^{k+1}=E Q^{k} .
\end{array} .\right.
$$

has a simple feedback :

$$
\left\{\begin{array}{l}
Q^{k+1}=C \otimes\left(A Q^{k}+B E Q^{k-1}+B W^{k}\right), \\
Z^{k+1}=E Q^{k} .
\end{array} .\right.
$$

The openloop dynamics corresponds to the system defined by :

$$
\bar{A}=A+B E, \bar{B}=B, \bar{C}=C, \bar{D}=\emptyset, \bar{E}=E, \bar{F}=\emptyset .
$$

\footnotetext{
${ }^{4}$ A better example would be $Z^{k}=E Q^{k}$, it could be dealt with an implicit version of systems studied here
} 
Nadir Farhi et al.

\subsection{Petri Nets with Consumption Arcs}

To increase the modeling power of Petri net it is useful to accept arcs with negative production or consuption arcs (negative entries in $H$ ). For example, let us suppose that from a place leaves two arcs it is useful to consider that one arc is leaving the place and the other arc is an input in the place with a negative production (each time the transition to which is connected this arc fires it consumes one token ${ }^{5}$. Therefore using this consumption arc we can resolve "choice" problem but new difficulties appears to prove the existence of stationary regimes because we can describe by this way more general dynamics.

Let us discuss a small example. The time variant Petri net in Figure-1-left has the dynamics :

$$
Z^{k+1}=\min \left\{V^{k+1}, a^{k}+Z^{k}\right\}, \text { with } a^{k}=\frac{(-1)^{k+1}+1}{2} .
$$

When $V^{k+1} \geq 1+Z^{k}$ for all $k$. This dynamics can be reduced to : $Z^{k+1}=a^{k}+Z^{k}$ which implies that $Z^{k+2}=1+Z^{k}=\min \left\{V^{k+2}, 1+Z^{k}\right\}$ which corresponds to the time-invariant Petri net of Figure-1-right. But there exist sequences of $V^{k}$ for which the sequences $Z^{k}$ are different whatever the initial conditions are. See the following sequences where we have denoted $\bar{Z}$ the sequence associated to the time invariant system and we have chosen the initial condition $\bar{Z}^{0}=Z^{0}$ and $\bar{Z}^{1}=Z^{1}$ :

\begin{tabular}{|c|lllllll|}
\hline$k$ & 0 & 1 & 2 & 3 & 4 & 5 & 6 \\
\hline$a^{k}$ & 0 & 1 & 0 & 1 & 0 & 1 & 0 \\
\hline$V^{k}$ & 0 & 0 & 1 & 1 & 1 & 2 & 2 \\
\hline$Z^{k}$ & 0 & 0 & 1 & 1 & 1 & 1 & 2 \\
\hline$Z^{k}$ & 0 & 0 & 1 & 1 & 1 & 2 & 2 \\
\hline
\end{tabular}
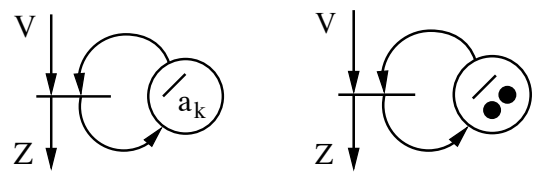

Fig. 1. Time variant (left) and time invariant (right) clock.

As soon as we accept consumption arcs it is possible to generate, by Petri net, non increasing sequences like $a^{k}$ as the difference of two increasing sequences.

\footnotetext{
${ }^{5}$ Input arc of a transition consume also tokens but they are different because they are combined with a minimum (they participate to the firing condition, the output arc of a transition with negative production consume tokens in the place where it is connected but it does not participate to the firing condition).
} 
The Petri net of Figure 3.3 has the dynamics :

$$
\left\{\begin{array}{l}
Q_{1}^{k+1}=1+Q_{2}^{k}, \quad Q_{1}^{0}=0, \\
Q_{2}^{k+1}=Q_{1}^{k}, \quad Q_{2}^{0}=0, \\
Z^{k+1}=\min \left\{V^{k+1}, Z^{k}+Q_{1}^{k}-Q_{2}^{k}\right\},
\end{array}\right.
$$

and we have

$$
Q_{1}^{k}-Q_{2}^{k}=\frac{(-1)^{k+1}+1}{2}=a^{k}
$$

Therefore it has an output equal to the one of time variant Petri net (Figure3.3-left). This kind of Petri net will be used to model traffic lights in the next section. In the following we will accept the consumption arcs which implies that
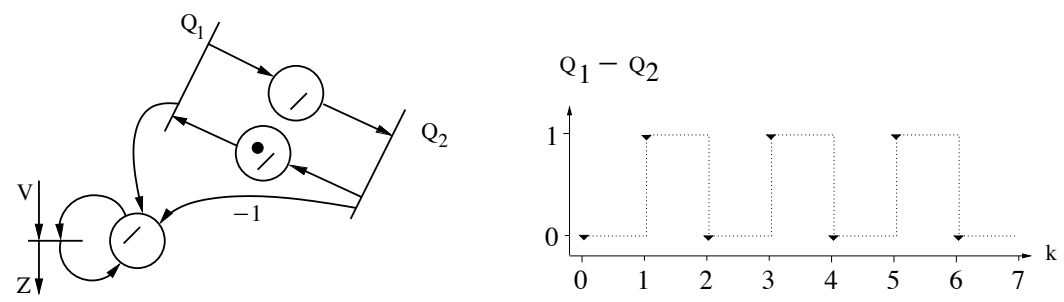

Fig. 2. Time invariant with consumption arc clock.

some entries of the matrices $A, B, E$ could be negative which introduce a lot of difficulties to study the existence of stationary regimes.

\section{Traffic modeling}

In this section, we build the Petri describing the traffic of a regular city. For that, we use three elementary Petri nets that we combine with the previous combinators. Then we use this Petri net to obtain the fundamental traffic diagram of a regular city which links the average flow to the density of vehicles.

First, let us give the assumptions done on the traffic in a regular city (Figure 3 ) is discribed as follows:

- Each road is made up of sections, which can contain only one vehicle.

- A crossing is a section with two possible outputs which can contain at most one vehicle.

- If a vehicle is not hampered by another one before, it remains one unit of time in a section, before moving.

- The vehicles move forward without overtaking, and at a crossing, one goes forward, the next on right or left depending of the crossing type.

- At each crossing there is a traffic light giving the moving authorizations. 
Nadir Farhi et al.

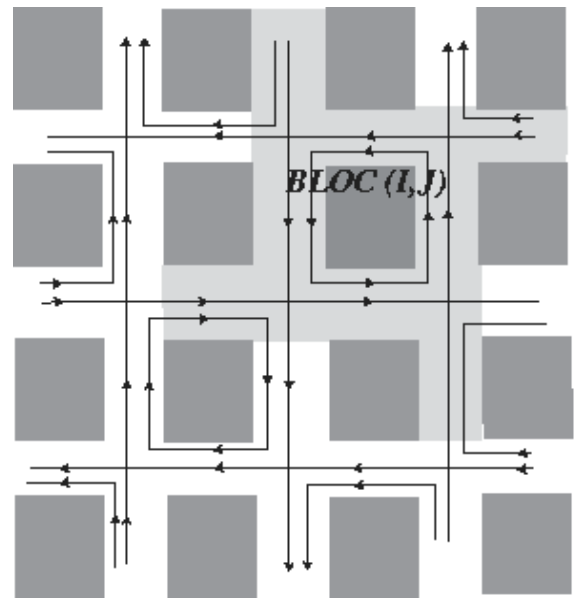

Fig. 3. A regular city.

\subsection{Two roads with one joint crossing}

Before building the Petri net of the complete regular town let us give the dynamic of a system composed of two circular road with one crossing and light control at the crossing (see Figure 4). A model of two circular roads with one crossing and right-priority has been presented in [2].

Each road is modelled by a set of sections. Each section is represented by two places $a$ and $\bar{a}$ in the Petri net. If $a=1$, the section is occupied by one vehicle and we have $\bar{a}=0$. If $a=0$, the section is free, and $\bar{a}=1$ gives the permission to enter in this section.
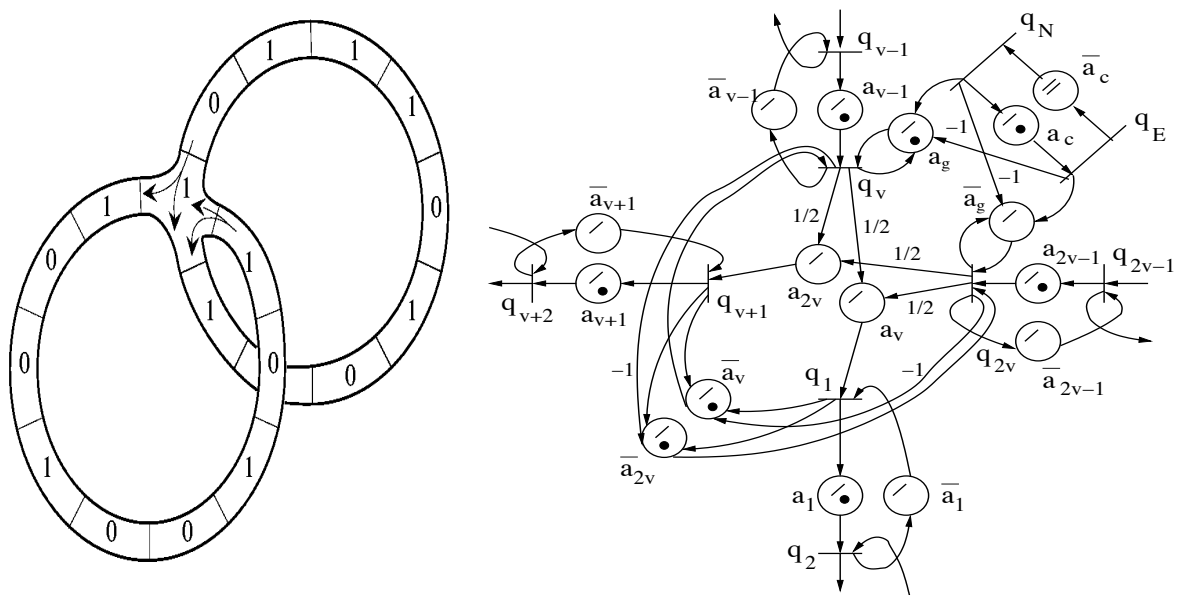

Fig. 4. Tow circular roads with one crossing managed by traffic lights. 
The traffic light is described in Figure 4 by the transition $q_{N}, q_{E}$ and the places connected to these transitions. We have, at the initial time, $a_{c}=a_{g}$ and $\bar{a}_{c}=\bar{a}_{g}=1-a_{c}$. If $a_{g}=1$, then $\bar{a}_{g}=0$, and the light is green for the vehicles coming from the north, red for the vehicles coming from the east. We assume that the phase times of the traffic light takes one time unit ${ }^{6}$. At the end of the first phase $q^{E}$ fires and consumes the token in $a_{c}$ and products one token in $\bar{a}_{c}$, one token in $\bar{a}_{g}$, and minus one token in $a_{g}$ (it consumes the token). The light commute to the other phase where $\bar{a}_{g}=\bar{a}_{c}=1$, which corresponds to a green light for the vehicles coming from the east, red light for the vehicles coming from the north. This phase takes also one unit of time, then $q^{N}$ fires and the system come back to the first phase.

Before entering in the crossing we have to know if it is free, this is done by the tokens in places $\bar{a}_{\nu}$ and $\bar{a}_{2 \nu}$ which must be counted correctly. The negative multiplicities is necessary for that. Thanks to the negative multiplicity each time a vehicle enter by the North (for example) the authorization by the east is consumed until the vehicle leaves the crossing. In Figure 4, when $a_{\nu}=1$ (resp. $a_{2 \nu}=1$ ), then the intersection is occupied by a vehicle which wants to go South (resp. West), and we have $\bar{a}_{\nu}=\bar{a}_{2 \nu}=0$. When $a_{\nu}=a_{2 \nu}=0$, the intersection is free, and $\bar{a}_{\nu}=\bar{a}_{2 \nu}=1$ gives the permission to enter into the crossing.

We consider that half of the vehicles entered in the crossing want go West (by entering in place $a_{2 \nu}$ ) and half want go South (by entering in place $a_{\nu}$ ). In fact, by rounding in appropriate way the number of vehicles in these two places, the even vehicles entered in the crossing go towards the South, and the odd ones go towards the West.

The dynamic of this system is then given by :

$$
\left\{\begin{array}{l}
Q_{i}^{k+1}=a_{i-1} Q_{i-1}^{k} \oplus \bar{a}_{i} Q_{i+1}^{k}, \quad i \in\{2, \ldots, \nu-1, \nu+2, \ldots, 2 \nu-1\}, \\
Q_{\nu}^{k+1}=\bar{a}_{\nu} Q_{1}^{k} Q_{\nu+1}^{k} / Q_{2 \nu}^{k} \oplus a_{\nu-1} Q_{\nu-1}^{k} \oplus a_{g} Q_{\nu}^{k} Q_{N}^{k} / Q_{E}^{k}, \\
Q_{2 \nu}^{k+1}=\bar{a}_{2 \nu} Q_{1}^{k} Q_{\nu+1}^{k} / Q_{\nu}^{k} \oplus a_{2 \nu-1} Q_{2 \nu-1}^{k} \oplus \bar{a}_{g} Q_{2 \nu}^{k} Q_{E}^{k} / Q_{N}^{k}, \\
Q_{1}^{k+1}=a_{\nu}\left\lfloor\sqrt{1 Q_{\nu}^{k} Q_{2 \nu}^{k}}\right\rfloor \oplus \bar{a}_{1} Q_{2}^{k}, \\
Q_{\nu+1}^{k+1}=a_{2 \nu}\left\lfloor\sqrt{Q_{\nu}^{k} Q_{2 \nu}^{k}}\right\rfloor \oplus \bar{a}^{\nu+1} Q_{\nu+2}^{k}, \\
Q_{N}^{k+1}=\bar{a}_{c} Q_{E}^{k}, \\
Q_{E}^{k+1}=a_{c} Q_{N}^{k} .
\end{array}\right.
$$

where $b / a$ means the largest $x$ such that $x \otimes a \leq b$, that is the standard subtraction when $b$ and $a$ are finite. Similarly, the square root minplus denoted by $\sqrt{ }^{\cdot}$ is the standard division by 2 , while $\lfloor\cdot\rfloor$ indicates the rounding down operator. For example Equation (4.1) is written in standard algebra as follows :

$$
Q_{1}^{k+1}=\min \left\{a_{\nu}+\left\lfloor\frac{1+Q_{\nu}^{k}+Q_{2 \nu}^{k}}{2}\right\rfloor, \bar{a}_{1}+Q_{2}^{k}\right\}
$$

\footnotetext{
${ }^{6}$ In fact the bar numbers in $a_{c}$ and $\bar{a}_{c}$.
} 


\subsection{Regular City Modeling.}

To model a regular city, we use the three elementary systems given in Figure 5 :

- A traffic section denoted by $\mathcal{T}_{Z \bar{Z}}^{V \bar{V}}(a, b)$,

- A crossing entry denoted by $\mathcal{E}_{Z Z_{s} Z_{w} Z_{0} Z_{0} Z_{1} Z_{2}}^{V V_{1} V_{2}}(a, b, c, d)$,

- A crossing exit denoted by $\mathcal{X}_{Z_{s} Z_{w} Z}^{V_{n} V_{e} V}(a, b)$,

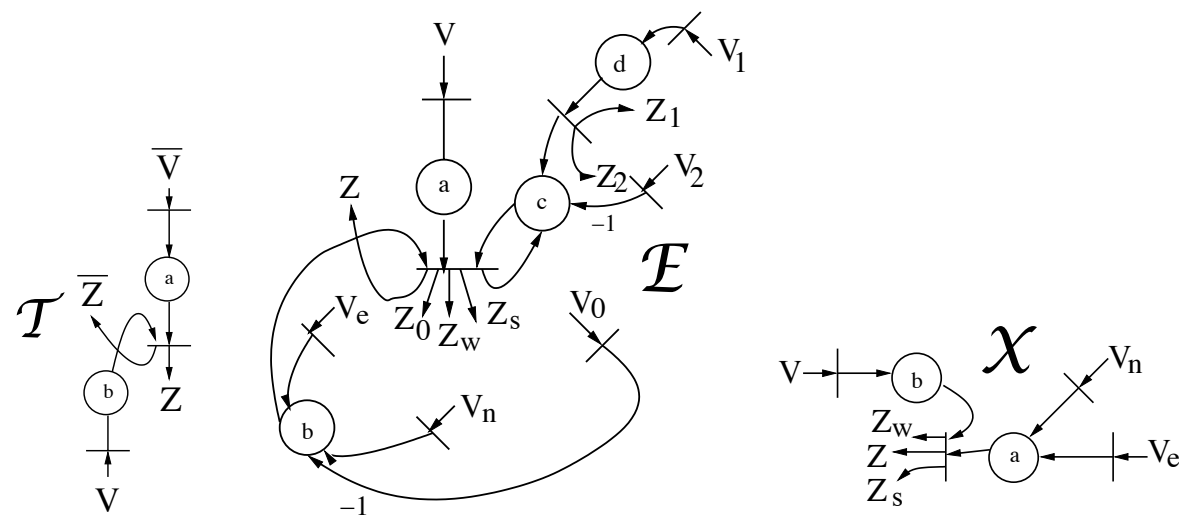

Fig. 5. Elementary systems

In the following, we give the construction of a regular city on a torus. That means that the East West roads and North South roads are circular and have been cut in Figure 3 representation.

To build the Petri Net we need only the three elementary systems that we compose with what we have called the composition operator.

A road with $n$ sections is defined by recurrence:

$$
{ }^{1} \mathcal{T}=\mathcal{T}, \quad{ }^{n} \mathcal{T}_{Z \bar{Z}}^{V \bar{V}}(a, c)={ }^{n-1} \mathcal{T}_{2 \bar{Z}}^{1 \bar{V}}(a, \bar{b}) \mathcal{T}_{Z 1}^{V 2}(b, c) .
$$

One road with $n-1$ places and one crossing is given by :

$\mathcal{R}_{Z \bar{Z} Z_{w} Z_{0} Z_{1} Z_{2} Z_{w}^{\prime}}^{V \bar{V} V_{e} V_{0} V_{1} V_{2} V^{\prime}}(a, d, e, f)={ }^{n-1} \mathcal{T}_{2 \bar{Z}}^{1 \bar{V}}(a, \bar{b}) \mathcal{E}_{14 Z_{w} Z_{0} Z_{1} Z_{2} Z_{2}}^{23 V_{0} V_{1} V_{2}}(b, \bar{c}, d, e) \mathcal{X}_{3 Z_{w}^{\prime} Z}^{4 V_{e}^{\prime} V}(c, f)$

A system of two roads with one joint crossing is given by:

$$
\mathcal{C}_{Z \bar{Z}}^{\bar{V} V \bar{V}^{\prime} \bar{Z}^{\prime} \bar{Z}^{\prime}}(a, b, e, f)=\mathcal{R}_{Z \bar{Z} 56789}^{\bar{V} V 01234}(a, b, c, d) \mathcal{R}_{Z^{\prime}}^{\bar{V}^{\prime} \bar{Z}^{\prime} 01234} V^{\prime}(e, \bar{b}, \bar{c}, f) .
$$

A block of four crossings as shown on Figure 6 is given by :

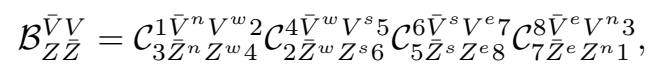




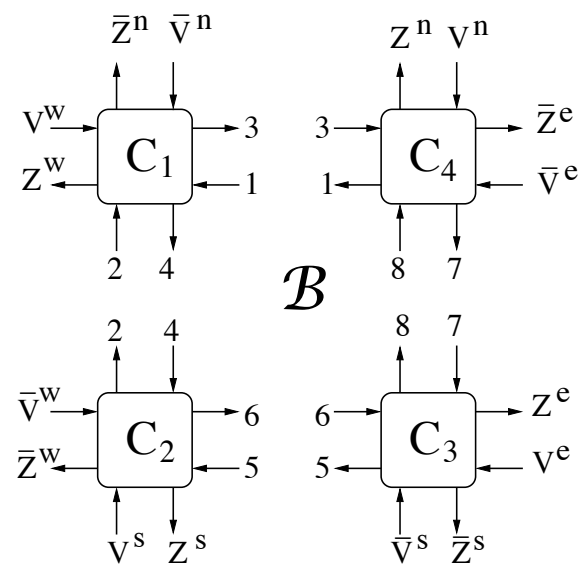

Fig. 6. One block of four crossings.

where we denote without index the vector of North West South East components for example $Z=\left(Z^{n}, Z^{w}, Z^{s}, Z^{e}\right)$.

Proceeding like above, we construct horizontal lines of blocks, then the regular city is the vertical contraction of lines of blocks. Finally the city on the torus Figure 7 is obtained by applying a feedback operation associated to the connection of the North input to the South output and the East in put to the West output.

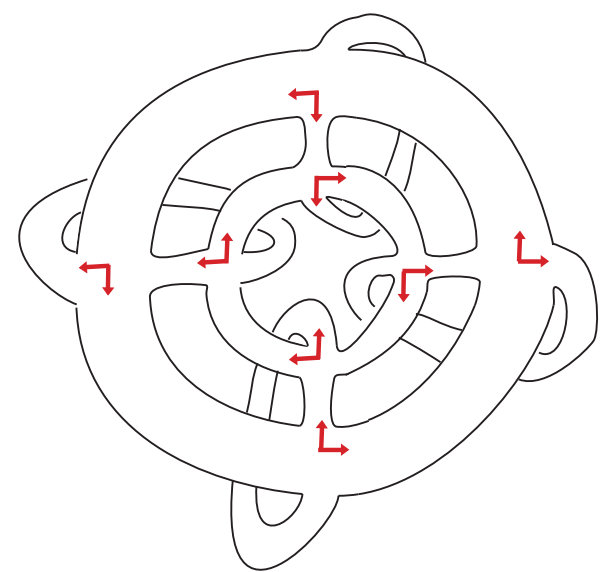

Fig. 7. Regular City on a Torus. 


\section{Fundamental Diagram and Stationary Regime}

\subsection{Fundamental Diagram}

By simulation of the Petri net, the fundamental traffic diagram of a regular city is obtained ${ }^{7}$, the density $d$ being the number of vehicles $p$ divided by the capacity of the city $m(d=p / m)$, and the flow $f$ being the average number of the vehicle displacements, given by: $f=f_{i}=\lim _{k \rightarrow+\infty} Q_{k}^{i} / k$ which is independent of transition $i$. The fundamental diagram is shown on Figure 8. This diagram present phases similar to the ones obtained on small systems [2,3]. See these references for discussion on the traffic interpretation of the different phases.

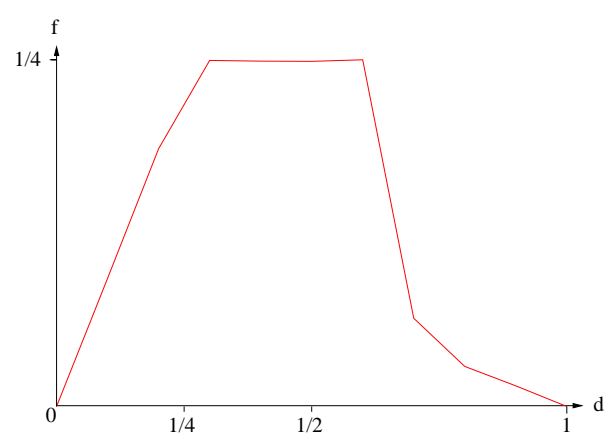

Fig. 8. The fundamental traffic diagram.

\subsection{Light Control feedback}

Let us discuss the significance of traffic light feedbacks for improving the traffic.

Given an open loop traffic light policy the vehicles reach a periodic regime which gives the average flow. This average flow does not depend ${ }^{8}$ on the initial position of the vehicles but the periodic regime may depends on it. This is shown on a small system composed of two roads with one crossing (the roads have the same size, and the routings and the green time durations being equidistributed).

Let us compare the periodic regime reached from an intial position using traffic lights, first in an open loop, and second in feedback on the number of vehicles in each road. In the feedback case, the controller compares the number of vehicles on each roads that it controls and gives more green light time to the road which has the largest vehicle number.

On Figure 9 and Tables 5.2 the letter $\mathrm{G}$ means green, $\mathrm{R}$ red for the corresponding traffic light $\mathrm{L}$. We conclude that even for a symmetrical system, there

\footnotetext{
7 The light phase time is chosen equal to two units of time for all crossings.

8 At least asymptotically on the scale of the system.
} 

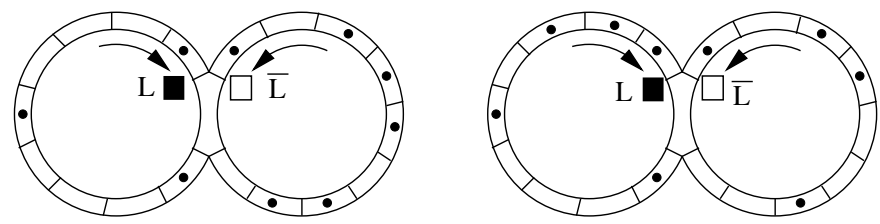

Fig. 9. Asymmetric (left) periodic regime, and symmetric one (right) on a symmetrical system.

\begin{tabular}{|c|c|c|}
\hline \begin{tabular}{l|l}
$\mathrm{k}$ & $\mathrm{I}$ \\
\end{tabular} & road I & road II \\
\hline \begin{tabular}{l|l}
0 & $C$ \\
\end{tabular} & $\begin{array}{llllllllll}1 & 0 & 0 & 0 & 1 & 0 & 0 & 0 & 1\end{array}$ & $\begin{array}{lllllllllll} & 0 & 1 & 1 & 0 & 1 & 1 & 1 & 0 & 1\end{array}$ \\
\hline 10 & $\begin{array}{llllllllll}0 & 1 & 0 & 0 & 0 & 1 & 0 & 0 & 0\end{array}$ & $\begin{array}{llllllllll}1 & 0 & 1 & 0 & 1 & 1 & 1 & 0 & 1 & 1\end{array}$ \\
\hline $2 \mathrm{~F}$ & $\begin{array}{llllllllll}0 & 0 & 1 & 0 & 0 & 0 & 1 & 0 & 0\end{array}$ & \begin{tabular}{l|lllllllll}
0 & 1 & 0 & 1 & 1 & 1 & 0 & 1 & 1 & 1
\end{tabular} \\
\hline $3 \mathrm{~F}$ & $\begin{array}{llllllllll}0 & 0 & 0 & 1 & 0 & 0 & 0 & 1 & 0\end{array}$ & \begin{tabular}{l|lllllllll}
1 & 0 & 1 & 1 & 1 & 0 & 1 & 1 & 1 & 0
\end{tabular} \\
\hline $4 \mathrm{C}$ & $\begin{array}{lllllllll}1 & 0 & 0 & 0 & 1 & 0 & 0 & 0 & 1\end{array}$ & $\begin{array}{lllllllllll}0 & 0 & 1 & 1 & 0 & 1 & 1 & 1 & 0 & 1\end{array}$ \\
\hline & mean $=5 / 2$ & mean $=6$ \\
\hline
\end{tabular}

\begin{tabular}{|c|c|c|c|}
\hline $\mathrm{k}$ & $\mathrm{L}$ & road I & road II \\
\hline 0 & $\mathrm{R}$ & $\begin{array}{llllllllll}1 & 0 & 0 & 0 & 1 & 0 & 0 & 0 & 1\end{array}$ & \begin{tabular}{l|llllllllll} 
& 0 & 0 & 1 & 1 & 0 & 1 & 1 & 1 & 0 & 1
\end{tabular} \\
\hline 1 & $\mathrm{R}$ & $\begin{array}{lllllllll}0 & 1 & 0 & 0 & 0 & 1 & 0 & 0 & 1\end{array}$ & $\begin{array}{llllllllll}1 & 0 & 1 & 0 & 1 & 1 & 1 & 0 & 1 & 0 \\
\end{array}$ \\
\hline 10 & $\mathrm{~K}$ & $\begin{array}{llllllllll}0 & 0 & 1 & 0 & 0 & 1 & 1 & 0 & 1\end{array}$ & $\begin{array}{lllllllllll}0 & 1 & 0 & 0 & 0 & 1 & 0 & 1 & 1 & 1\end{array}$ \\
\hline 11 & & $\begin{array}{lllllllll}0 & 0 & 0 & 1 & 0 & 1 & 0 & 1 & 1\end{array}$ & \begin{tabular}{l|lllllllll}
1 & 0 & 1 & 0 & 0 & 0 & 1 & 1 & 1 & 0
\end{tabular} \\
\hline 12 & & $\begin{array}{lllllllll}1 & 0 & 0 & 0 & 1 & 0 & 1 & 1 & 1\end{array}$ & $\begin{array}{lllllllllll}0 & 0 & 0 & 1 & 0 & 0 & 1 & 1 & 0 & 1 \\
\end{array}$ \\
\hline & & mean $=17 / 4$ & mean $=17 / 4$ \\
\hline
\end{tabular}

Table 1. Asymmetric (left) and symmetric (right) periodic regimes.

exist initial distributions for which the vehicles are not distributed in a uniform way in the periodic regime but using a stabilizing feedback this uniform distribution can be obtained. This is also observed for the complete regular city (Figure $10)$.
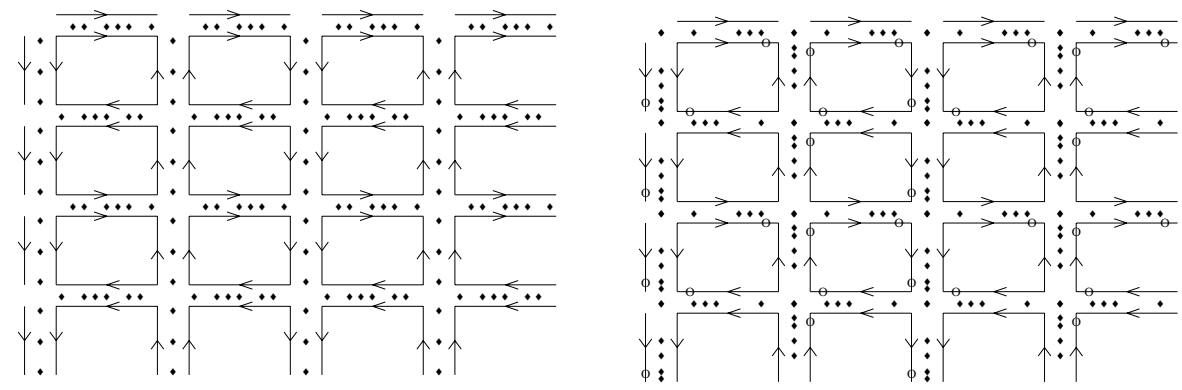

Fig. 10. Periodic Regime : - open loop lights (left), - feedback lights (right).

\section{Conclusion.}

We have built a traffic model of regular cities with traffic lights using inputoutput Petri nets with negative multiplicities. The construction is based on a 
system theory where Petri nets are represented by matrices defined on a mixed algebra (some components live in the standard algebra the other ones in the minplus algebra). The calculus is not associative but the standard combinators (parallel series feedback composition) can be easily defined.

Using this construction the fundamental diagram of regular city can be computed and the interest of the feedback traffic light control can be shown.

\section{References}

1. F. Baccelli, G. Cohen, G.J. Olsder, and J.P. Quadrat : Synchronization and Linearity, Wiley, 1992 available http://maxplus.org.

2. N. Farhi, M. Goursat, and J.P. Quadrat : Derivation of the fundamental traffic diagram for two circular roads and a crossing using minplus algebra and Petri net modeling, In Proceedings of the 44th IEEE - CDC-ECC Seville December 2005.

3. N. Farhi, M. Goursat, and J.P. Quadrat: Fundamental Traffic Diagrams of Elementary Road Networks, to appear.

4. B. Derrida, M.R. Evans, Exact steady state properties of the one dimensional asymmetric exclusion model pp. 1-16 in Probability and Phase Transition ed G. Grimmett, Kluwer Ac. Pub. 1994

5. C. Diadaki, M. Papageorgiou, K. Aboudolas, A Multivariable regulator approach to traffic-responsive network-wide signal control Control Eng. Practice N. 10, pp. 183-195, 2002.

6. E. Mancinelli, Guy Cohen, S. Gaubert, J.-P. Quadrat, E. Rofman : On Traffic Light Control of Regular Towns INRIA Report Sept. 20001.

7. S. Gaubert : Théorie des systèmes linéaires dans les dioides, Thesis dissertation, École des Mines de Paris, (1992).

8. T. Murata Petri Nets: Properties, Analysis and Applications Proceedings of the IEEE, Vol. 77, No. 4, pp. 541-580, 1989.

9. P. Lotito, E. Mancinelli, J.P. Quadrat A Min-Plus Derivation of the Fundamental Car-Traffic Law, IEEE-AC V.50, N.5, pp.699-705, 2005.

10. D. Chowdhury, L. Santen, A. Shadschneider Statistical physics of vehicular traffic and some related systems. Physics Report 329, pp. 199-329, 2000

11. E. Brokfeld, R. Barlovic, A. Schadschneider, M. Schreckenberg, Optimizing traffic lights in a cellular automaton model for city traffic, Physical Review E, volume 64, 2001.

12. Cécile Appert and Ludger Santen Modélisation du trafic routier par des automates cellulaires, Actes INRETS 100, Ecole d'automne de Modlisation du Trafic Automobile 2002.

13. T. Ushio On controllability of controlled Petri nets, Control-theory and advanced technology, Vol. 5, No 3, pp. 265-275, September 1989.

14. Fukui M., Ishibashi Y., Phase Diagram on the Crossroad II: the Cases of Different Velocities, Journal of the Physical Society of Japan, Vol. 70, N. 12, pp. 3747-3750, 2001.

15. Fukui M., Ishibashi Y., Phase Diagram on the Crossroad, Journal of the Physical Society of Japan, Vol. 70, N. 9, pp. 2793-2797, 2001.

16. Fukui M., Ishibashi Y., Phase Diagram for the traffic on Two One-dimensional Roads with a Crossing, Journal of the Physical Society of Japan, Vol. 65, N. 9, pp. 2793-2795, 1996.

17. http://scilabsoft.inria.fr 